\title{
Analyzing the behavior of a porous nano- hydroxyapatite/polyamide 66 (n-HA/PA66) composite for healing of bone defects
}

This article was published in the following Dove Press journal:

International Journal of Nanomedicine

13 January 2014

Number of times this article has been viewed

Yan Xiong'
Cheng Ren'
Bin Zhang'
Hongsheng Yang'
Yun Lang'
Li Min'
Wenli Zhang'
Fuxing Pei'
Yonggang Yan'
Hong Li'
Anchun Mo3
Chongqi Tu'
Hong Duan'
'Department of Orthopedics, West
China Hospital, ${ }^{2}$ School of Physical
Science and Technology, ${ }^{3}$ Department
of Oral Implant, West China College
of Stomatology, Sichuan University,
Chengdu, People's Republic of China

Correspondence: Hong Duan; Chongqi Tu Department of Orthopedics, West China Hospital, Sichuan University, No 37 Guo Xue Lane, Chengdu, Sichuan 610064, People's Republic of China Tel +8628 85422570

Fax +86 2885422570

Email duanhong1970@।26.com; tuchongqitucq@।63.com
Abstract: The aim of this study was to analyze the behavior of the porous nano-hydroxyapatite/ polyamide 66 (n-HA/PA66) composite grafted for bone defect repair through a series of biological safety experiments, animal experiments, and a more than 5-year long clinical follow-up. The biological safety experiments, carried out in accordance with the Chinese Guo Biao and Tolerancing (GB/T)16886 and GB/T16175, revealed that porous n-HA/PA66 composite had no cytotoxicity, no sensitization effect, no pyrogenic reaction, and that its hemolysis rate was $0.59 \%$ (less than $5 \%$ ). Rabbit models of tibia defects with grafted porous n-HA/PA66 composite were established. After 2 weeks, the experiment showed that osteogenesis was detected in the porous n-HA/PA66 composite; the density of new bone formation was similar to the surrounding host bone at 12 weeks. After 26 weeks, the artificial bone rebuilt to lamellar bone completely. In the clinical study, a retrospective review was carried out for 21 patients who underwent serial radiographic assessment after treatment with porous n-HA/PA66 composite grafts following bone tumor resection. All wounds healed to grade A. No postoperative infections, delayed deep infection, nonspecific inflammation, rejection, or fractures were encountered. At a mean follow-up of 5.3 years, the mean Musculoskeletal Tumor Society's (MSTS) 93 score was 29.3 points (range: 28-30 points) and mean radiopaque density ratio was $0.77 \pm 0.10$. The radiologic analysis showed that porous n-HA/PA66 composite had been completely incorporated with the host bone about 1.5 years later. In conclusion, this study indicated that the porous n-HA/PA66 composite had biological safety, and good biocompatibility, osteoinduction, and osseointegration. Thus, the porous n-HA/PA66 composite is an ideal artificial bone substitute and worthy of promotion in the field.

Keywords: biomaterial, artificial bone materials, porous n-HA/PA66 composite (n-HA/PA66), bone defects healing, bone grafting

\section{Introduction}

Bone is both an important tissue and a major organ of the human body. Among other things, bones are important for support, protection, loading, hematopoiesis, and calcium storage. Bone defects are quite common clinically. The treatment of large bone defects caused by trauma, infection, and tumor excision represents a great challenge in the clinical practice setting. Bone grafting - which includes autografts, allografts, and synthetic substitutes - is an important treatment for large bone defects. ${ }^{1,2}$ Successful incorporation of grafted material into bone defects depends on the material's ability to promote new bone formation and provide a scaffold for osteogenesis. As is well known, autogenous bone grafting is the gold standard in the reconstruction of bone defects; it has the advantage of providing both bone stock and osteogenic precursor cells. Due to limited resources, the autograft technique presents difficulties in meeting the demands 
for large bone defects. Because of rejection and the spreading of infection diseases, allograft use is restricted in clinical practice. Hence, synthetic bone substitutes are being increasingly used in bone reconstruction techniques. ${ }^{3}$ Searching for the ideal artificial bone substitute for repairing bone defects is therefore an important subject in orthopedics.

While the use of biological materials has long been studied, the use of artificial bone materials to repair bone defects emerged in the 1980 s and has developed rapidly since. ${ }^{4}$ The basic concept of artificial bone material is that it is used as a bone tissue substitute, wherein it is incorporated into the bone defect to reestablish bone clinically. Searching for the ideal artificial bone material is presently a hot topic in orthopedics. The properties of the ideal artificial bone materials include biological safety, fine biocompatibility, good absorbability and biodegradability, good porous structure (an average pore diameter 200-400 microns), good mechanical properties, osteogenesis, osteoinduction, and osteoconduction. ${ }^{5,6}$ Currently, the artificial biomaterials used as bone tissue substitutes mainly include artificially-synthesized materials and materials derived from natural biological organisms. Amongst other materials, artificially-synthesized materials contain calcium phosphate ceramic, calcium phosphate cement (CPC), bioactive glass, polyamide (PA), polylactic acid (PLA/PLLA), and polyglycolic acid (PGA). Materials derived from natural biological organisms include mainly nano-hydroxyapatite (n-HA), collagen, chitins, coral bone (CAB), and natural bone. ${ }^{1-3}$

Because it is difficult to prepare an ideal bone tissue substitute with a single material, many studies have being carried out in recent years looking at developing bone substitute composites for use in repairing bone defects. ${ }^{7-9}$ A novel biomedical composite, porous nano-hydroxyapatite/polyamide 66 (n-HA/PA66), has been developed in recent years. ${ }^{7-9}$
This composite has good similarity to human bone; that is, n-HA has a similar composition and crystal structure to the mineral of natural bone. n-HA, which has good biocompatibility and bone regeneration, can prompt injured bone to reproduce. ${ }^{9}$ PA66, which is a kind of high molecular synthetic material with sufficient resources, acts in the role of a collagen matrix in the composite, avoiding the immunogenicity problem of natural polymeric materials. The polar amide bond, carboxyl group, and carboxyl group of PA66 can grow, form osteoids, and mineralize tissue cell to promote healing of bone defects. Moreover, this composite has good mechanical performance and can serve as a load-bearing bone substitute; for example, its dense composite has been extensively used in repairing vertebral plates and has proven to have a good effect on bone reconstruction. ${ }^{10}$

\section{Materials and methods Biological safety experiments}

The porous n-HA/PA66 composite was prepared by n-HA (molecular weight of 1,000; nanoparticle diameter of 80-100 nm) and PA66 (molecular weight of 18,000-20,000), which were provided by the Sichuan National Nanotechnology Co, Ltd (Chengdu, People's Republic of China). The size of the porous n-HA/PA66 composite sample was $4 \times 2 \times 2 \mathrm{~mm}^{3}$. The average pore size was 500 microns, with a porosity of $75 \%-85 \%$, and a compressive strength of 2-10 $\mathrm{mPa}$ (Figure 1). The porous n-HA/PA66 composite was synthesized using chemical foaming techniques, as previously described in other studies. ${ }^{7-9}$

To evaluate the biological safety of the porous n-HA/PA66 composite, a cytotoxicity test, sensitivity test, pyrogen test, and a hemolysis test were carried out according to the Chinese Guo Biao and Tolerancing (GB/T)16886 and GB/T16175. ${ }^{11}$ The extract liquid of the porous n-HA/PA66 composite was prepared
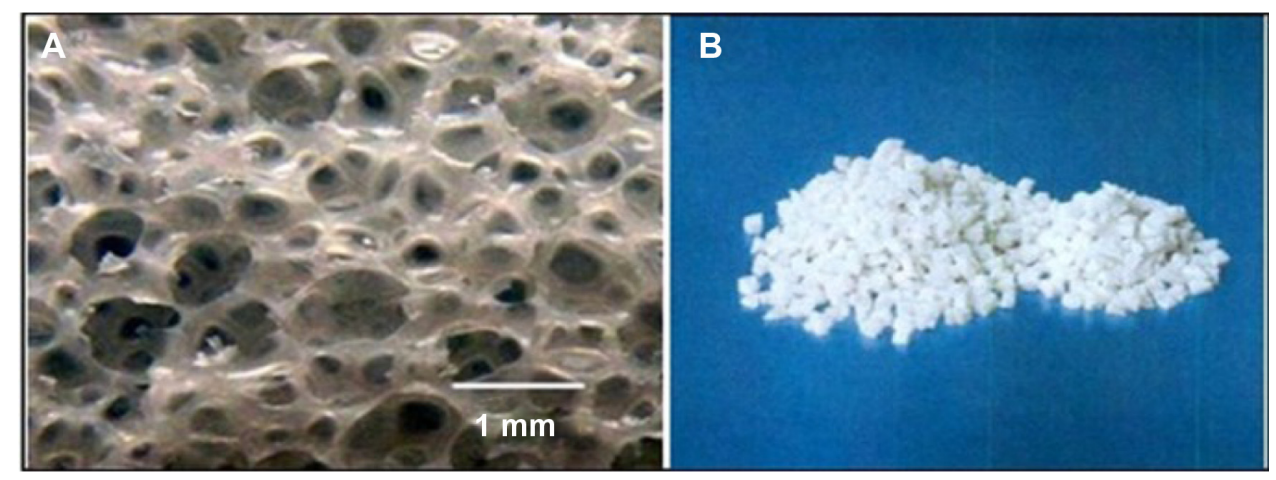

Figure I The morphology of porous n-HA/PA66 composite.

Notes: (A) A porosity of 75\%-85\% and an average pore size of 500 microns; (B) A size of $4 \times 2 \times 2 \mathrm{~mm}^{3}$.

Abbreviation: n-HA/PA66, nano-hydroxyapatite/polyamide 66 . 
in biological safety experiments. A mouse fibroblast L929 cell strain, which was inoculated into cell culture dish, was prepared for the cytotoxicity test. The experimental group was given porous n-HA/PA66 extract liquid and the control group received sterile physiological saline. All dishes were put into an incubator $\left(37^{\circ} \mathrm{C}, 5 \%\right.$ carbon dioxide $\left.\left[\mathrm{CO}_{2}\right]\right)$ for 24 hours to observe the cytolysis ratio. In the sensitization test, the red punctuation and hydropsia of guinea pigs were observed at 24 hours, 48 hours, and 72 hours by subcutaneously injecting porous n-HA/PA66 extract liquid into the back of the guinea pigs. The control group was injected with sterile physiological saline. The extract liquid was injected via rabbit ear vein for the pyrogen test. The temperature changes were measured before and after the injection. The hemolysis test was conducted on anticoagulated fresh rabbit blood. The experimental group received extract liquid and the control group sterile physiological saline.

\section{Animal implantation studies}

In the animal implantation study, 16 New Zealand White rabbits $(2.0-2.5 \mathrm{~kg}$, randomly male and female, offered by Sichuan University, West China Experimental Animal Center) received tibia bone defects $4 \mathrm{~mm}$ in diameter. Performed as a self-contrast in paired design, the right rabbit tibia was grafted with porous n-HA/PA66 composite, while the left tibia (control) of the same rabbit was grafted with autogenous iliac bone. For both groups, we performed macroscopic observations and radiological, histological, and scanning electron microscope analysis at 2, 4, 12, and 26 weeks after surgery.

\section{Clinical studies}

From November 2007 to July 2008, there were 21 consecutive patients who fulfilled the following inclusion criteria: 1) benign bone tumor confirmed by pathology; 2) their treatment consisted of one operation and single implantation of the porous n-HA/ PA66 composite; 3 ) there was complete follow-up data; and 4) the patient showed no recurrence. We excluded a woman who suffered from giant cell tumor of the right proximal tibia, where the tumor had shown recurrence after 2 years of follow-up.

The clinical studies group included nine male and twelve female patients. Their age at the time of surgery was 18-56 years, with a mean age of 33 years. Eight lesions were located in the diaphysis, twelve in the metaphysis of a long bone, and only one in a short bone. Histological examinations revealed that nine were giant cell tumor, six were bone cysts, and six were fibrous dysplasia. The size of the lesions ranged from $4.0 \times 3.0 \times 3.0 \mathrm{~cm}^{3}$ to $17.0 \times 3.0 \times 3.0 \mathrm{~cm}^{3}$, and no case suffered from pathological fracture. All cases underwent curettage, were inactivated, and then the tumor cavities filled with porous n-HA/PA66 composite. While four cases received internal fixation implants, six were implanted with both variant bone plates and internal fixation. The implant amount of porous n-HA/PA66 composite ranged from 10-30 g, with an average of $20 \mathrm{~g}$. One, 3, 6, 9, and 12-month follow-up was observed after treatment, and after we recommended an inspection be carried out each year (Table 1).

The clinical evaluation included objective findings focusing on the operative incision's healing, blood tests, biochemical assays, and subjective complaints measured with the Musculoskeletal Tumor Society's (MSTS) 93 limb functional recovery score system (upper limb: pain, adjacent joint function, emotional acceptance, supports, positioning of hand, lifting ability; lower limb: pain, adjacent joint function, emotional acceptance, supports, walking ability, gait; each $0-5$ points, total of 30 points). ${ }^{12}$

Using standard projections and evaluated independently, the radiographs were analyzed by three investigators, and the data were checked for interobserver agreement. In the event of differences, the patient's radiograph was reevaluated by all three observers together. The radiographic integration of porous n-HA/PA66 composite was assessed according to the Lane and Sandhu radiological criteria system, to determine the presence of the distinct bridging trabeculae at the recipient bone-implant interface and also the amount of incorporation of porous n-HA/PA66 composite. ${ }^{13}$

A software program (Syngo version V35; Siemens Medical Systems, Erlangen, Germany) for quantitative analysis was used to measure the X-ray radiopaque density value. An area of interest was determined over the grafted section (see Figure 2) with number of counts recorded. An identical area of interest was determined over a non-lesion bone area near the operated section, and the grafting-to-non-lesion count ratio (G/N ratio) was then calculated (see Figure 2). The normal value of the $\mathrm{G} / \mathrm{N}$ ratio was 1.00 . After curettage of the bone tumor and implantation of the porous n-HA/PA66 composites, patients were followed-up for between 5-5.5 years (5.3 years on average) by panoramic X-ray and computed tomography (CT) plain scan, and spiral CT 3D reconstruction.

For statistical analysis, SPSS 17.0 (IBM Corporation, Armonk, NY, USA) software was used. The values are presented as mean \pm standard deviation (SD). One-way analysis of variance (ANOVA) was used to determine differences between the radiopaque density ratios for dependent variables measured at the normal level (sex, diagnosis, location, bone grafted mass). The correlation was analyzed, with linear correlation used to determine the relationship between radiopaque 
Table I Details of patients after implantation of the porous n-HA/PA66 composite

\begin{tabular}{|c|c|c|c|c|c|c|c|c|c|}
\hline Number & Age & Sex & Diagnosis & Location & $\begin{array}{l}\text { Bone } \\
\text { grafting }\end{array}$ & Follow-up & MSTS & Radiographic finding & $\begin{array}{l}\mathbf{G} / \mathbf{N} \\
\text { ratio }\end{array}$ \\
\hline 1 & 37 & $\mathrm{~F}$ & FD & Right proximal femur & $30 \mathrm{~g}$ & 5.2 years & 29 & Complete incorporation & 0.82 \\
\hline 2 & 20 & $\mathrm{~F}$ & $\mathrm{BC}$ & Left heel bone & $10 \mathrm{~g}$ & 5.5 years & 30 & Complete incorporation & 0.41 \\
\hline 3 & 18 & M & $\mathrm{BC}$ & Right proximal femur & $25 \mathrm{~g}$ & 5.5 years & 29 & Complete incorporation & 0.75 \\
\hline 4 & 27 & M & GCT & Left distal femur & $25 \mathrm{~g}$ & 5.4 years & 29 & Complete incorporation & 0.74 \\
\hline 5 & 34 & $\mathrm{~F}$ & FD & Right femur & $25 \mathrm{~g}$ & 5.1 years & 30 & Complete incorporation & 0.82 \\
\hline 6 & 49 & $\mathrm{~F}$ & FD & Right proximal tibia & $10 \mathrm{~g}$ & 5 years & 29 & Complete incorporation & 0.75 \\
\hline 7 & 36 & $\mathrm{~F}$ & $\mathrm{BC}$ & Right proximal humerus & $20 \mathrm{~g}$ & 5.2 years & 29 & Complete incorporation & 0.79 \\
\hline 8 & 21 & M & FD & Left proximal femur & $20 \mathrm{~g}$ & 5.5 years & 30 & Complete incorporation & 0.78 \\
\hline 9 & 28 & M & $\mathrm{BC}$ & Right proximal tibia & $30 \mathrm{~g}$ & 5.5 years & 28 & Complete incorporation & 0.78 \\
\hline 10 & 21 & $\mathrm{~F}$ & GCT & Right proximal femur & $30 \mathrm{~g}$ & 5 years & 29 & Complete incorporation & 0.77 \\
\hline 11 & 24 & $\mathrm{~F}$ & FD & Left distal femur & $20 \mathrm{~g}$ & 5.2 years & 29 & Complete incorporation & 0.62 \\
\hline 12 & 39 & M & GCT & Left proximal femur & $20 \mathrm{~g}$ & 5.3 years & 29 & Complete incorporation & 0.78 \\
\hline 13 & 49 & M & GCT & Left distal femur & $15 \mathrm{~g}$ & 5.5 years & 30 & Complete incorporation & 0.75 \\
\hline 14 & 18 & $\mathrm{~F}$ & $\mathrm{BC}$ & Right proximal femur & $20 \mathrm{~g}$ & 5 years & 30 & Complete incorporation & 0.84 \\
\hline 15 & 23 & $\mathrm{~F}$ & $\mathrm{BC}$ & Right distal femur & $20 \mathrm{~g}$ & 5.2 years & 30 & Complete incorporation & 0.79 \\
\hline 16 & 31 & M & GCT & Right proximal tibia & $10 \mathrm{~g}$ & 5.3 years & 30 & Complete incorporation & 0.92 \\
\hline 17 & 43 & M & GCT & Right proximal tibia & $20 \mathrm{~g}$ & 5 years & 29 & Complete incorporation & 0.88 \\
\hline 18 & 56 & $\mathrm{~F}$ & GCT & Right proximal femur & $30 \mathrm{~g}$ & 5.4 years & 29 & Complete incorporation & 0.86 \\
\hline 19 & 47 & $\mathrm{~F}$ & GCT & Right distal femur & $15 \mathrm{~g}$ & 5.4 years & 29 & Complete incorporation & 0.78 \\
\hline 20 & 55 & $\mathrm{~F}$ & GCT & Left proximal humerus & $20 \mathrm{~g}$ & 5.5 years & 28 & Complete incorporation & 0.70 \\
\hline 21 & 20 & M & FD & Right proximal femur & $25 \mathrm{~g}$ & 5.1 years & 30 & Complete incorporation & 0.83 \\
\hline
\end{tabular}

Abbreviations: n-HA/PA66, nano-hydroxyapatite/polyamide 66; F, female; M, male; FD, fibrous dysplasia of bone; G/N ratio, grafting-to-non-lesion ratio; BC, bone cyst; GCT, giant cell tumor; MSTS, Musculoskeletal Tumor Society score.

density ratios and the age of patients at the time of surgery. A $P$-value of less than 0.05 was considered significant.

\section{Results}

\section{Biological safety experiments}

For the cytotoxicity test, the cytolysis ratio of the experimental group was found to be $0(\mathrm{R}=0)$. There was no significant difference between the experimental group and the control group. In the sensitization test, the erythema and edema failed to be found in the guinea pigs injected by leaching liquor and saline. In the pyrogen test, the temperatures of

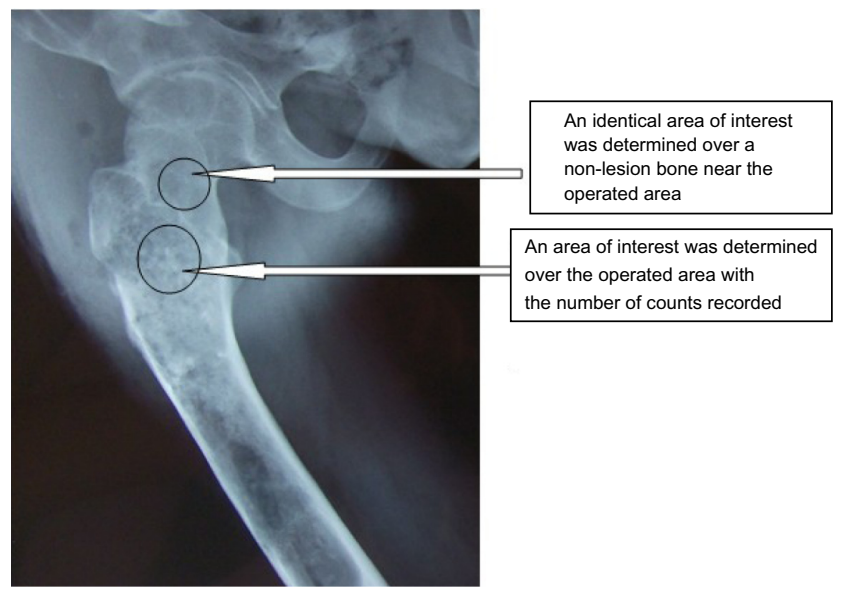

Figure 2 Determination of the grafting-to-non-lesion count ratio ( $\mathrm{G} / \mathrm{N}$ ratio). experimental rabbits rose by $0.6^{\circ} \mathrm{C}$, which met the requirements of the pyrogen test. In the hemolysis test, the results showed that the hemolysis rate was $0.59 \%$ (less than $5 \%$ ). Therefore, the porous n-HA/PA66 composite had no cytotoxicity, no sensitization effect, no pyrogenic reactions, and the hemolysis rate was $0.59 \%$ (less than $5 \%$ ), which met biological safety standards according to the Chinese GB/ T16886 and GB/T16175.

\section{Animal implantation studies}

Macroscopic observation showed that there was no postoperative infection or material displacement. Histological assessment indicated that osseous tissue gradually crept into the interconnected porosity and connected with the material directly, without fibrous tissue. The amount of new bone and calcium crystals were increased at 4-8 weeks, and the new bones continuously matured. After 12 weeks, new bone formed in the pores, gradually linked with each other, and connected with materials directly. After 26 weeks, the interconnected porosity was completely filled with new bone tissue, integrated into a whole, and showed good osteoinductivity and osteocompatibility. Marrow cavity was also generated at that time (Figures 3 and 4). Panoramic X-ray analysis showed that by 26 weeks the test side had been repaired completely, the density of which was equivalent to the normal control. 


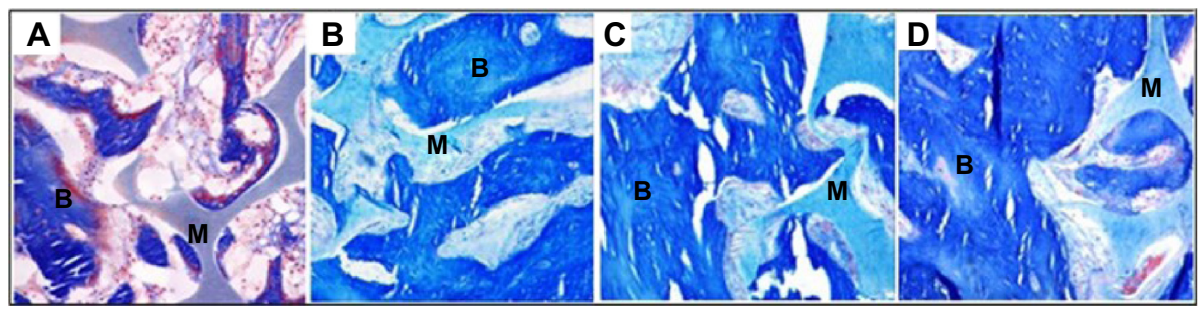

Figure 3 Figure showing how the amount of new bone and calcium crystals increased as the time goes on.

Notes: Bone and calcium crystals at (A) 2 weeks, (B) 8 weeks, (C) 12 weeks, and (D) 26 weeks. Masson staining was used, x200 magnification. After 26 weeks, interconnected porosity was filled completely with new bone tissue, which integrated into a whole. Abbreviations: $M$, material; $B$, bone.

\section{Clinical studies}

All cases were followed-up for 5-5.5 years postoperatively (average of 5.3 years). The results were satisfactory. There was nothing obviously exceptional in the $\mathrm{C}$-reactive protein, routine blood investigations, biochemical tests, and hemagglutination inspections of all patients. All wounds healed to grade A. No postoperative infections, delayed deep infection, nonspecific inflammation, rejection, or fractures were encountered. Limb function was good and so was joint motion near the tumor spots. In addition, no patient complained of pain. The internal steel plates were still rigidly fixed and no joint surface subsidence occurred. The mean MSTS score was 29.3 points (range: $28-30$ points).

Radiographs which were obtained immediately after surgery revealed radiolucent zones between the implanted porous n-HA/PA66 composite and the surrounding bone. Periodic assessments showed that $25 \%$ of grafting zones were replaced by new bones in 2-3 months; after 6-9 months, new bone formation rose to $50 \%$. The defects were fully occupied by new bone within 1.5 years (Figures 5 and 6 ). Radiographic observations analyzed the grafting and non-lesion areas for differences. Mean radiopaque density ratio was $0.77 \pm 0.10$ after grafting with porous n-HA/PA66. This was most obvious in cancellous bone such as the heel bone $(\mathrm{G} / \mathrm{N}=0.41)$. No degenerative changes were observed in adjacent joints. After implantation with porous n-HA/PA66, the variables were evaluated; there was no relationship between age, sex, diagnosis, bone graft mass, and radiopaque density ratio (Table 2). We documented a significant difference in the radiopaque density between metaphyseal and diaphyseal location of grafting $(F=9.65, P=0.01)$. Mean radiopaque density ratio was $0.67 \pm 0.10$ after implantation with porous n-HA/PA66 in the metaphyseal region, and $0.85 \pm 0.03$ in the diaphyseal region. As shown in Table 1, the $\mathrm{G} / \mathrm{N}$ ratio was $<1$. Because new bone growth in the grafting zone blocked the $\mathrm{X}$-ray transmission, as time went on, the more new bones grew, the smaller the $\mathrm{G} / \mathrm{N}$ value, and this value became stationary at about 1.5 years. Due to new bone tissue growing more numerously and faster in the metaphysis, the $\mathrm{G} / \mathrm{N}$ ratio curve was lower and steeper than the curve for diaphysis (Figure 7).

The excluded female patient previously mentioned suffered from a giant cell tumor of the right proximal tibia and the tumor had shown recurrence after follow-up for 2 years. She underwent a second operation and the resected tumor tissues were sent for pathology inspection. The result showed that the pores of porous n-HA/PA66 composite were filled with new bone and tumor tissues which integrated into a whole (Figure 8).

\section{Discussion}

\section{Biological safety}

Biomaterials are ones which can be implanted into the body and joined with tissues and organs together. Biological safety

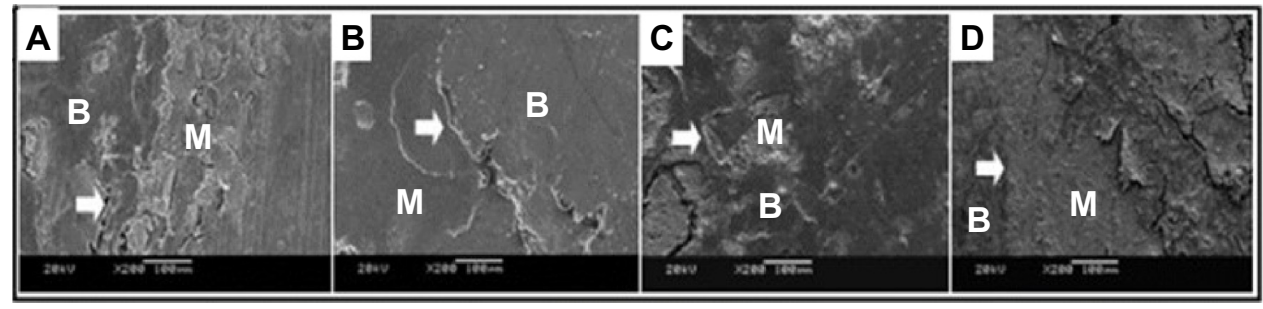

Figure 4 The SEM images $(\times 2,000$ magnification) of the porous n-HA/PA66 composite with implantation into the rabbit tibia.

Notes: The interface of material and bone at (A) 2 weeks, (B) 8 weeks, (C) 12 weeks, and (D) 26 weeks. After 26 weeks, the porous n-HA/PA66 composites integrated with host bone completely.

Abbreviations: SEM, scanning electron microscope; n-HA/PA66, nano-hydroxyapatite/polyamide 66; M, material; B, bone. 

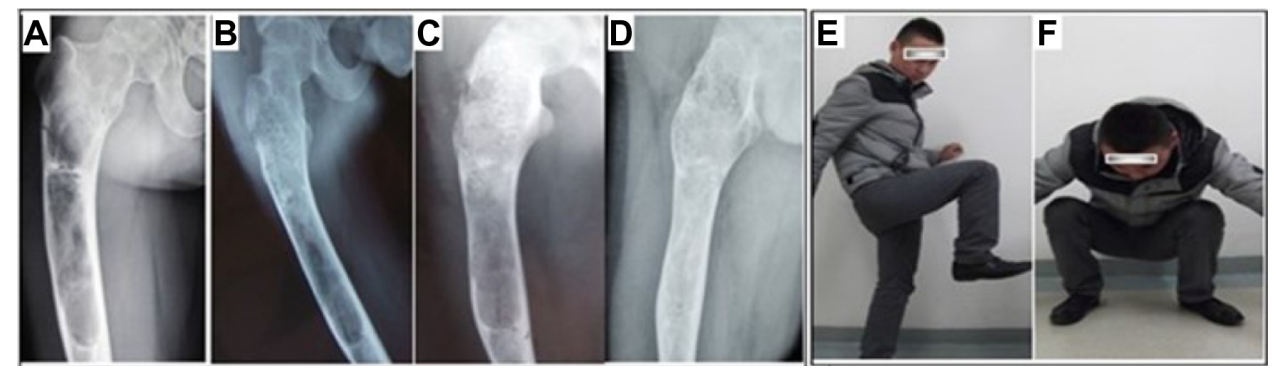

Figure 5 A 20-year-old man suffering from fibrous dysplasia of the right proximal femur.

Notes: (A) The original image of the defect site. Periodic radiological assessments showed (B) during the period of 6 months, $50 \%$ of the grafting zones were replaced by new bones, and (C) fully occupied at 1.5 years after surgery. (D) With a more than 5 years follow-up, the porous n-HA/PA66 composite had been completely incorporated with the host bone. (E and $\mathbf{F}$ ) grafting-to-non-lesion count ratio was 0.83 , the patient was satisfied with the result, and the MSTS was 30 .

Abbreviations: n-HA/PA66, nano-hydroxyapatite/polyamide 66; MSTS, Musculoskeletal Tumor Society score.

is one of the most important factors when dealing with biomedical materials. ${ }^{14}$ Any new biomedical materials in the early stages of clinical application must be tested according to ISO standards or the appropriate standards of the jurisdiction where it is being used. Laboratory and animal experiments are the main methods for evaluating the biological safety of biomedical materials. In our studies, biological safety experiments showed that the porous n-HA/PA66 composite had no cytotoxicity, no sensitization effect, and no pyrogenic reactions. Further, the hemolysis rate was $0.59 \%$, which met biological safety standards according to the Chinese GB/ T16886 and GB/T16175. In animal implantation studies, there was no suppuration or necrosis after grafting the porous n-HA/PA66, and new bone formation rebuilt to lamellar bone completely after 26 weeks. In clinical studies, all wounds healed to grade A. All patients healed well, experiencing no infection, aseptic inflammation, or rejection reactions. None of the patients had serious side effects such as functional damage of the liver and kidney. Therefore, it is evident that the treatment of large bone defects by grafting porous n-HA/ PA66 composite is secure.

\section{Biocompatibility}

Biocompatibility is another important factor in the study of biomedical material intended for use in issue or organ repair. ${ }^{15}$ The previous study showed that porous n-HA/PA66 had biomimetic and porous characteristics, representing good biocompatibility for healing bone defects. ${ }^{8}$

Bone can be considered as a nanocomposite material made up of collagen protein fibers threading through a HA mineral phase, which make up about $60-70 \mathrm{wt} \%$ of the total bone tissue. Based on a biomimetic mechanism, we prepared n-HA/PA66 composite that had similar composition or molecular groups to that of natural bone; the composite had an n-HA:PA66 ratio of 6:4. ${ }^{9}$ The organic PA66 macromolecular network uniformly covered the nano-sized HA granules, similar to the interactions that exist between the components of normal bone. Electrostatic forces and hydrogen bonds existed between the ions and groups in the composite material; these included interactions between the $\mathrm{Ca}^{2+}$ and $\mathrm{PO}_{4}^{3-}$ charged groups of $\mathrm{HA}$ and the $-\mathrm{C}-\mathrm{O}$ and $-\mathrm{NH}-$ groups in PA66. Additionally, coordination bonds were formed between the $-\mathrm{COO}$ group of PA66 and $\mathrm{Ca}^{2+}$ group of n-HA. Therefore, the high propor-
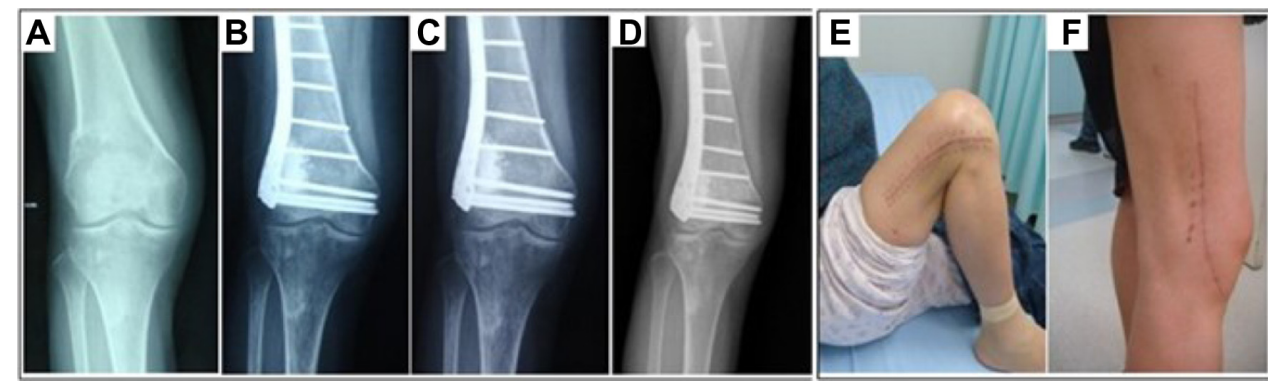

Figure 6 Images of a 47-year-old woman treated by a giant cell tumor resection and fixed with an internal steel plate after the porous n-HA/PA66 composite grafted in the right distal femur.

Notes: Periodic radiological assessments showed that the new bones developed and the porous n-HA/PA66 composite incorporated with the host bone gradually. The internal steel plate was still rigidly fixed and no joint surface subsidence occurred. (A) Preoperative; (B) a half-year after resection; (C) I year; and (D) 5 years later. The grafting-to-non-lesion count ratio was 0.78 and the MSTS score was 29 after 5 years follow-up (E and $\mathbf{F}$ ).

Abbreviations: n-HA/PA66, nano-hydroxyapatite/polyamide 66; MSTS, Musculoskeletal Tumor Society score. 
Table 2 Statistical data on 21 patients

\begin{tabular}{|c|c|c|c|c|}
\hline Variable & $\begin{array}{l}\text { Total } \\
(\mathrm{N}=\mathbf{2} \mathrm{I})\end{array}$ & G/N ratio & $\boldsymbol{F}$ & $P$ \\
\hline $\mathrm{Age}^{\mathrm{a}}$ & 21 & $0.77 \pm 0.10$ & 0.87 & 0.36 \\
\hline \multicolumn{5}{|l|}{ Sex ${ }^{b}$} \\
\hline Female & 12 & $0.75 \pm 0.12$ & 1.42 & 0.33 \\
\hline Male & 9 & $0.80 \pm 0.06$ & & \\
\hline Diagnosis $^{b}$ & & & 0.83 & 0.45 \\
\hline GCT & 9 & $0.80 \pm 0.07$ & & \\
\hline FD & 6 & $0.77 \pm 0.08$ & & \\
\hline$B C$ & 6 & $0.73 \pm 0.16$ & & \\
\hline Location $^{\mathrm{b}}$ & & & 9.65 & 0.01 \\
\hline Metaphysis & 12 & $0.67 \pm 0.10$ & & \\
\hline Diaphysis & 8 & $0.85 \pm 0.03$ & & \\
\hline Bone grafted mass ${ }^{b}$ & & & 1.02 & 0.33 \\
\hline$\leq 20 \mathrm{~g}$ & 13 & $0.73 \pm 0.16$ & & \\
\hline$>20 \mathrm{~g}$ & 8 & $0.78 \pm 0.07$ & & \\
\hline
\end{tabular}

Notes: aCorrelation between the two variables was analyzed with linear correlation; bone-way analysis of variance (ANOVA).

Abbreviations: $\mathrm{G} / \mathrm{N}$ ratio, grafting-to-non-lesion ratio; $\mathrm{FD}$, fibrous dysplasia of bone; BC, bone cyst; GCT, giant cell tumor.

tion of HA and the uniform distribution of n-HA granules in the PA66 matrix enable the scaffolding material to possess good biocompatibility, high bioactivity, and enough mechanical strength. ${ }^{7,16,17}$ Previous studies have confirmed that an n-HA/PA66 composite with an n-HA:PA66 ratio of 6:4 had more biocompatibility than that of other ratios. ${ }^{9}$

An appropriate pore diameter and porosity make a biomaterial more biocompatible. The current research shows that pores of 100-500 microns are suitable for bone tissue growth. ${ }^{18}$ Hulbert et al reported 100 microns was the minimum pore diameter to allow bone tissue growth, and 150 microns the ideal. ${ }^{19}$ Vaz et al showed that 500 microns was the most appropriate for bone growth. ${ }^{20}$ In fact, the human bone is a relatively loose complex structure. The structure of bone consists of dense tissue at the outermost layer, changing gradually through semi-dense to a loose mesh structure in the innermost layer. Therefore, when the porous n-HA/PA66 composite was grafted, the pores of the host bone and the grafted material gradually integrated into the two cross-linked form, which would be able to maintain normal physiological metabolism in the healing of the bone's defects. In our clinical studies, a significant difference was documented in the radiopaque density between the metaphysis and diaphysis of porous n-HA/PA66 composite filling $(F=9.65, P=0.01)$. The pores of metaphyseal bone and materials were better integrated because of the loose mesh structure of the metaphysis. This mesh structure increased the localized supply of nutrition and blood, and as a result new bones grew more numerously and faster; the $\mathrm{G} / \mathrm{N}$ ratio curve of the metaphysis was therefore lower and steeper (Figure 6). Additionally, porosity is another important indicator of a porous mesh structure. The mutual penetration and the size of the aperture are the most important factors in bone regeneration. The higher porosity materials can provide a better channel structure. ${ }^{21-23}$ Jie and Yubao showed that when porosity of the porous n-HA/PA66 composite was more than $50 \%$, there was a positive effect on cell adherence and there were extracellular matrix deposits on the surface. ${ }^{24}$ Higher porosity is also advantageous to the transmission of oxygen and nutrients and to the growth of blood vessels and nerves. Thus, good conditions were provided for new bone formation. However, the increase in pore

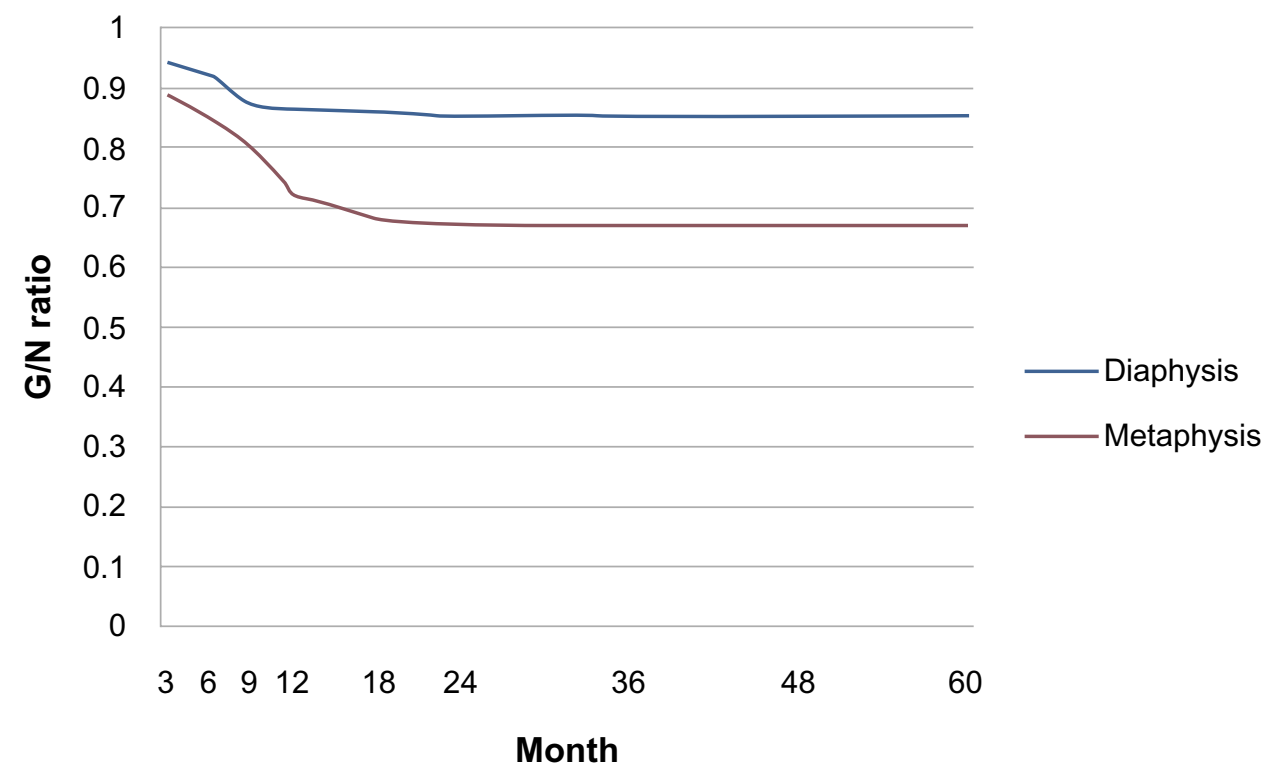

Figure 7 Comparing the G/N ratio between the metaphyseal and diaphyseal areas where the porous n-HA/PA66 composites were implanted after bone tumor resection. Abbreviations: G/N ratio, grafting-to-non-lesion ratio; n-HA/PA66, nano-hydroxyapatite/polyamide 66. 


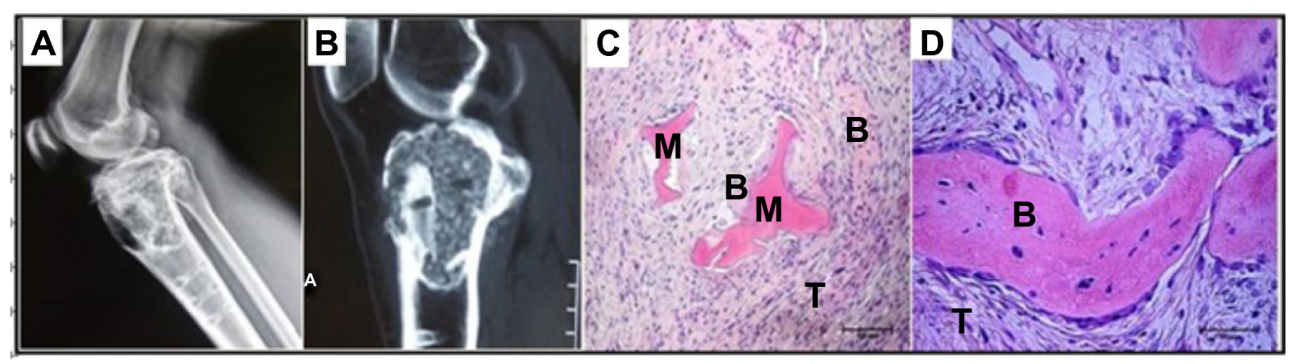

Figure 8 Images of the female patient that did not meet inclusion criteria due to recurring giant cell tumor.

Notes: A woman, 46 years, suffered from giant cell tumor of the right proximal tibia and had shown recurrence after a follow-up of 2 years. She received treatment by tumor resection and single implantation of the porous composite. (A and $\mathbf{B})$ show the $\mathrm{X}$-ray image and magnetic resonance image of GCT recurrence; (C and $\mathbf{D})$ show the histological images of GCT recurrence, (C) $\mathrm{HE} \times 100$, (D) $\mathrm{HE} \times 200$ ).

Abbreviations: n-HA/PA66, nano-hydroxyapatite/polyamide 66; GCT, giant cell tumor; M, material; B, bone; T, tumor; HE, hematoxylin and eosin staining.

size and porosity decreases the material's mechanical strength. Therefore, a compromise should be reached between material properties and mechanical strength to satisfy bone conduction requirements. In previous studies, porous n-HA/PA66 composite with an average pore diameter of 500 microns, porosity of $75 \%-85 \%$, and a compression strength of $2-10 \mathrm{MPa}$ (similar to human cancellous bone) satisfied the requirements to repair bone defects. ${ }^{8,9,17}$

\section{Osteoinduction and osseointegration}

The beginning of bone formation and bone cell proliferation is a very complex process. Some research has found that three-dimensional pore structures could facilitate cell adhesion, differentiation, and proliferation, and help with fibrovascular and nerve colonization, so that the new bone "crawls" into the material to achieve early osteoinduction. ${ }^{8,9}$ Moreover, the osteoinduction ability of the n-HA/PA66 composite may be associated with a "nanometer-sized effect". The surface of a nano-material is significantly larger than a micron-material, and the number of atoms are significantly increased on the surface. Thereby, owing to their greater surface area, the nano-sized particles are more biologically active compared with micron-sized particles. The previous studies have shown that micron-HA only had osteoconduction without osteoinduction, yet nano-HA had both. ${ }^{7,9} \mathrm{Kim}$ et al reported that nano-HA particles which were compounded into the polypropylene matrix have good biological activity, and that their osteoinduction was superior to ordinary HA particles. ${ }^{25}$ In earlier studies where porous n-HA/PA66 composite was implanted into the deep layer of the back muscles of rabbits, newly formed bone tissue was found in pores after 26 weeks. ${ }^{26}$ This proved that the porous n-HA/PA66 composite had good osteoinductivity.

Artificial bone substitutes for bone defect repair not only have good osteoinductivity, they can also integrate with host bone so that the implanted materials can play a good physiological function for a long time. The ideal bone substitute material can bond to osseous tissue tightly. Rosen et al have indicated that it was easy to cause loosening (leading to failure) if the fibrous tissue growth wrapped the interface of the bone substitutes after implantation..$^{27}$ In our studies, new bone formed in the pores and connected with materials directly. Radiographic observations demonstrated that the grafting and non-lesion area combined as a whole. Owing to good osseointegration of the porous n-HA/PA66 composite, no case showed joint collapse or internal fixation loosening during the average 5.3 years of follow-up. The pathological results of the excluded female patient showed pores were filled with new bones and tumor tissues, which integrated with the porous n-HA/PA66 composite.

\section{Clinical application}

According to research into biomaterials, there are various artificial bone substitute materials, including biomedical metal materials, polymer materials, and some biological ceramic materials. The results of clinical applications of artificial bone substitute materials are mixed. ${ }^{1-3,5,28-32}$ Demineralized bone matrix (DBM) can impart more osteoinductive potential than standard mineralized allograft. However, variable clinical response is not for structural supports. Synthetic ceramics which are without infectious or immunogenic potential are known to be osteoconductive but lack intrinsic osteogenic potential, as is found with autograft. CPC remains inert for long periods with insubstantial resorption. However, the initial liquid state can lead to difficulties during placement, such as extrusions into intra-articular regions. Collagen/ceramic composites (eg, n-HA/PE, collagen) which have been found to be effective autograft extenders, have osteoconduction and osteoinduction properties. n-HA/PA66 has been found to have biological safety, good biocompatibility, and osteoinduction 
and osseointegration in in vitro and in vivo studies, and has been shown to be a prospective application in the clinical setting. ${ }^{33,34}$

In our clinical studies, all cases underwent curettage, inactivation, and were filled with porous n-HA/PA66 composite. Solid fusion was obtained in the 6 months after the operation. Thus, the porous n-HA/PA66 composite can be effectively used for repairing bone defects caused by benign bone cysts and tumor-like lesions. Additionally, in clinical practice, the porous n-HA/PA66 composite was used to repair bone defects in limb bone fractures, aseptic necrosis of bone, arthrodesis, and orthopedics of grafted bone, with good effects produced. According to our studies, the porous n-HA/PA66 composite implanted into both metaphyseal and diaphysic defects and achieved positive results; MSTS scores also revealed favorable functional outcomes (mean: 29.3 points). However, porous n-HA/PA66 is more suitable for filling the diaphyseal and short bone defects $(P=0.01)$.

After the tumor is resected and the bone grafts adequately, different fixations are used according to the different nature of the lesion: 1) the porous n-HA/PA66 composite is grafted alone with no internal fixation when lesion involves the bone cross-sectional area $<50 \% ; 2$ ) the lesion involves a bone cross-sectional area $>50 \%$, or the diameter of cortical bone defect is $<50 \%$ with risk of pathologic fracture, so a steel plate is internally fixed after the porous n-HA/PA66 composite is grafted; and 3 ) the lesion involves a bone crosssectional area $>50 \%$, the diameter of cortical bone defect is $>5 \mathrm{~cm}$, or pathologic fractures have occurred, so an allogeneic cortex bone plate is tightly embedded in the window of the lesion cortex bone and a parallel plate is fixed at the same time. Owing to the appropriate procedures and repair methods being carried out, no patients saw lesion fractures and were healed satisfactorily in our studies. After surgery, all patients should accept the functional training and regular re-examination schedule which involves blood tests and imaging examination (panoramic radiography, $\mathrm{CT}$ plain scan, and spiral CT $3 \mathrm{D}$ reconstruction) to assess any changes in the graft and lesion healing. After the lesion heals well for about 2years, the internal fixation is considered for removal.

\section{Conclusion}

In conclusion, the porous n-HA/PA66 composite has biological safety, good biocompatibility, osteoinduction and osseointegration; it is an efficient, safe, and highly costeffective bone substitute material worthy of promotion in the orthopedic field.

\section{Acknowledgment}

The work described in this paper was supported by the National Science and Technology Support Program of the People's Republic of China (2007BAE131304).

\section{Disclosure}

The authors report no conflicts of interest in this work.

\section{References}

1. Giannoudis PV, Dinopoulos H, Tsiridis E. Bone substitutes: an update. Injury. 2005;36(Supp1 3):S20-S27.

2. Moore WR, Graves SE, Bain GI. Synthetic bone graft substitutes. ANZ J Surg. 2001;71(6):354-361.

3. Kaveh K, Ibrahim R, Bakar MZA, Ibrahim TA. Bone grafting and bone graft substitutes. J Anim Vet Adv. 2010;9(6):1055-1067.

4. Ratner B, Hoffman AS, Schoen FI, Lemons JE. Biomaterials Science: an Introduction to Materials in Medicine. San Diego: Academic Press; 2004:162-164.

5. Costantino PD, Friedman CD. Synthetic bone graft substitutes Otolaryngol Clin North Am. 1994;27(5):1037-1074.

6. Cypher TJ, Grossman JP. Biological principles of bone graft healing. $J$ Foot Ankle Surg. 1996;35(5):413-417.

7. Wang X, Li Y, Wei J, de Groot K. Development of biomimetic nano-hydroxyapatite/poly (hexamethylene adipamide) composites Biomaterials. 2002;23(24):4787-4791.

8. Wang H, Li Y, Zuo Y, Li J, Ma S, Cheng L. Biocompatibility and osteogenesis of biomimetic nano-hydroxyapatite/polyamide composite scaffolds for bone tissue engineering. Biomaterials. 2007;28(22): 3338-3348.

9. Yang AP, Li H, Li JD, Li YB, Yan YG, Xiang HZ. Fabrication of porous n-HA/PA66 composite for bone repair. Key Engineering Materials. 2007;330:321-324

10. Yang X, Song Y, Liu L, Liu H, Zeng J, Pei F. Anterior reconstruction with nano-hydroxyapatite/polyamide-66 cage after thoracic and lumbar corpectomy. Orthopedics. 2012;35(1):66-73.

11. Li H, Li Y, Yan Y, Zhou G, Wang M, Cheng L. [Preparation and biological safety evaluation of porous n-HA/PA66 composite]. Sheng Wu Yi Xue Gong Cheng Xue Za Zhi. 2008;25(5):1126-1129. Chinese.

12. Enneking WF, Dunham W, Gebhardt MC, Malawar W, Fritchard DJ. A system for the functional evaluation of reconstructive procedures after surgical treatment of tumors of the musculoskeletal system. Clin Orthop Relat Res. 1993;(286):241-246.

13. Lane JM, Sandhu HS. Current approaches to experimental bone grafting. The Orthop Clin North Am. 1987;18(2):213-225.

14. Tsuchiya T. Studies on the standardization of cytotoxicity tests and new standard reference materials useful for evaluating the safety of biomaterials. J Biomater Appl. 1994;9(2):138-157.

15. Black J. Biological Performance of Materials: Fundamentals of Biocompatibility. Boca Raton: CRC Press; 2006.

16. Zhang X, LiY, Lv G, Zuo Y, MuY. Thermal and crystallization studies of nano-hydroxyapatite reinforced polyamide 66 biocomposites. Polymer Degradation and Stability. 2006;91(5):1202-1207.

17. Wu L, LiYB, Zhang L, Yang WH, ZuoY, MuYH. Study on the Biomimetic Properties of Bone Substitute Material: Nanohydroxyapatite/Polyamide 66 Composite. Materials Science Forum. 2006;510-511:938-941.

18. Green D, Walsh D, Mann S, Oreffo RO. The potential of biomimesis in bone tissue engineering: lessons from the design and synthesis of invertebrate skeletons. Bone. 2002;30(6):810-815.

19. Hulbert SF, Morrison SJ, Klawitter JJ. Tissue reaction to three ceramics of porous and non-porous structures. J Biomed Mater Res. 1972;6(5):347-374. 
20. Vaz L, Lopes AB, Almeida M. Porosity control of hydroxyapatite implants. J Mater Sci Mater Med. 1999;10(4):239-242.

21. Chang BS, Lee CK, Hong KS, et al. Osteoconduction at porous hydroxyapatite with various pore configurations. Biomaterials. 2000;21(12):1291-1298.

22. Liu DM. Preparation and characterisation of porous hydroxyapatite bioceramic via a slip-casting route. Ceramics International. 1998;24(6): 441-446.

23. Milosevski M, Bossert J, Milosevski D, Gruzvska N. Preparation and properties of dense and porous calcium phosphate. Ceramics International. 1999;25(8):693-696.

24. Jie W, Yubao L. Tissue engineering scaffold material of nano-apatite crystals and polyamide composite. European Polymer Journal. 2004;40(3):509-515.

25. Kim HM, Furuya T, Kokubo T, Miyazaki T, Nakamura T. Composition of apatite produced in simulated body fluids. Key Engineering Materials. 2001;218-220:621-624.

26. Xu Q, Lu H, Zhang J, Lu G, Deng Z, Mo A. Tissue engineering scaffold material of porous nanohydroxyapatite/polyamide 66. Int $J$ Nanomedicine. 2010;5:331-335.
27. Rosen PS, Reynolds MA, Bowers GM. The treatment of intrabony defects with bone grafts. Periodontol. 2000;22(1):88-103.

28. Fleming JE, Cornell CN, Muschler GF. Bone cells and matrices in orthopedic tissue engineering. Orthop Clin North Am. 2000;31(4): 357-374.

29. Lane JM, Tomin E, Bostrom MPG. Biosynthetic bone grafting. Clin Orthop Relat Res. 1999;(Suppl 367):S107-S117.

30. Vaccaro AR. The role of the osteoconductive scaffold in synthetic bone graft. Orthopedics. 2002;25(Suppl 5):s571-s578.

31. Murugan R, Ramakrishna S. Development of nanocomposites for bone grafting. Composites Science and Technology. 2005;65(15): 2385-2406

32. Morrison R. Bone Grafts and Bone-Graft Substitutes[M]//Manual of Spine Surgery. Springer Berlin Heidelberg. 2012:75-80.

33. Li J, Man Y, Zuo Y, et al. In Vitro and In Vivo Evaluation of a nHA/PA66 Composite Membrane for Guided Bone Regeneration. J Biomater Sci Polym Ed. 2010

34. Su B, Peng X, Jiang D, et al. In Vitro and In Vivo Evaluations of NanoHydroxyapatite/Polyamide 66/Glass Fibre (n-HA/PA66/GF) as a Novel Bioactive Bone Screw. PLoS One. 2013;8(7):e68342.
International Journal of Nanomedicine

\section{Publish your work in this journal}

The International Journal of Nanomedicine is an international, peerreviewed journal focusing on the application of nanotechnology in diagnostics, therapeutics, and drug delivery systems throughout the biomedical field. This journal is indexed on PubMed Central, MedLine, CAS, SciSearch $\AA$, Current Contents ${ }^{\circledR} /$ Clinical Medicine,

\section{Dovepress}

Journal Citation Reports/Science Edition, EMBase, Scopus and the Elsevier Bibliographic databases. The manuscript management system is completely online and includes a very quick and fair peer-review system, which is all easy to use. Visit http://www.dovepress.com/ testimonials.php to read real quotes from published authors. 